Mens

Revue d'histoire intellectuelle et culturelle

\title{
Le collège classique comme lieu de mémoire : présences contemporaines d'une formation disparue
}

\section{Louise Bienvenue}

Volume 13, numéro 2, printemps 2013

URI : https://id.erudit.org/iderudit/1025981ar

DOI : https://doi.org/10.7202/1025981ar

Aller au sommaire du numéro

Éditeur(s)

Centre de recherche en civilisation canadienne-française

ISSN

1492-8647 (imprimé)

1927-9299 (numérique)

Découvrir la revue

Citer cet article

Bienvenue, L. (2013). Le collège classique comme lieu de mémoire : présences contemporaines d'une formation disparue. Mens, 13(2), 7-34.

https://doi.org/10.7202/1025981ar d'utilisation que vous pouvez consulter en ligne. 


\title{
Perspectives
}

\section{Le collège classique comme lieu de mémoire : présences contemporaines d'une formation disparue ${ }^{1}$}

\author{
Louise Bienvenue \\ Université de Sherbrooke
}

Cinquante ans après son arrêt de mort, signé par le rapport Parent et le gouvernement Lesage, le cours classique n'est pas tout à fait enterré. Son spectre plane toujours sur la société québécoise. Par «la volonté des hommes " et "le travail du temps ", selon les termes consacrés par Pierre Nora, ce fleuron du système éducatif d'avant la Révolution tranquille semble avoir acquis un véritable statut de lieu de mémoire; il s’impose comme " point de cristallisation de l'héritage collectif $^{2}$ ».

\footnotetext{
${ }^{1}$ Cette réflexion a d'abord été présentée dans le cadre du congrès de la Société du patrimoine politique du Québec (SOPPOQ), tenu le 18 octobre 2013 sur le thème "Les années 1950 au Québec ». L'auteure aimerait remercier Pierre Meese pour son assistance dans le travail de recherche documentaire de même que le Conseil de recherches en sciences humaines du Canada pour son soutien financier.

2 Cité dans Alexandre Lafon, "Les Lieux de mémoire : un livre, un concept, des utilisations possibles ", [En ligne], [http://crdp.ac-bordeaux.fr/cddp47/ mediatheque/intro_casseneuil.asp] (10 décembre 2013). Voir aussi Pierre Nora, "Entre mémoire et histoire, la problématique des lieux de mémoire ", dans Pierre Nora (dir.), Les lieux de mémoire, t. 1 : La République : symboles, monuments, pédagogie, commémorations, contre-mémoire, Paris, Gallimard, 1984, p. XVII-XLII.
} 
Cette étude vise à explorer en quoi le cours classique et les institutions qui l'ont dispensé au fil des ans travaillent toujours l'inconscient scolaire québécois d'une manière discrète, certes, et néanmoins persistante. En effet, la référence au cours classique est encore invoquée de nos jours dès que vient le temps de réfléchir collectivement au rapport que nous entretenons au savoir et à la culture. Davantage que d'autres archétypes mémoriels comme l'école de rang ou l'institut familial, le collège classique - pourtant fréquenté historiquement par une minorité de garçons et un nombre presque anecdotique de filles ${ }^{3}$ - est l'institution qui incarne au mieux un " ancien temps " de l'éducation, mais aussi l'envers de la doxa pédagogique actuelle, axée sur la démocratisation du savoir, l'expression de soi, la perspective utilitariste de même que la mixité des sexes et des cultures. Dans la trame de notre mémoire culturelle, il y aurait donc un avant et un après au cours classique.

Notre réflexion sera structurée en trois temps. En recourant à la notion de tradition inventée ${ }^{4}$, nous tenterons, dans la première partie, de départager le collège historique du collège imaginé. Nous y expliquerons en quoi l'image classique du collège, invoquée dans les discours, n'a rien d'intemporel et se rapporte plus précisément à l'expérience des années d'après-guerre, période qui marque le zénith de l'institution. Dans la deuxième partie, nous brosserons un portrait succinct de la variété des manifestations mémorielles contemporaines qui conservent vivant le cours classique et qui en font une référence toujours active de notre vie sociétale. Enfin, d'une manière plus ciblée,

3 En 1953, chez les francophones du Québec, $8 \%$ des fils et moins de $1 \%$ des filles étaient inscrits au secondaire. Andrée Lévesque, "La Révolution tranquille : une histoire réinterprétée et genrée ", Possibles, vol. 35, n 1 (automne 2011), p. 12-18, [En ligne], [http://redtac.org/possibles/files/2011/11/Possibles-RevTranquillevol35-Final1.pdf]. Pour étayer son analyse, l'auteure se réfere à Claude Galarneau, Les collèges classiques au Canada français, Montréal, Éditions Fides, 1978, p. 63-65.

${ }^{4}$ Une notion initialement développée dans le cadre des travaux sous la direction d'Eric J. Hobsbawm et Terence Ranger, The Invention of Tradition, Cambridge, Cambridge University Press, 1983. 
nous nous intéresserons aux usages du cours classique dans le débat sur l'éducation des vingt dernières années au Québec.

\section{Le collège " classique ": une tradition partiellement inventée}

Le collège classique, comme le rappelait récemment l'historienne Denyse Baillargeon, est " une institution quasi mythique au Québec ». Il demeure dans l'imaginaire collectif comme ce lieu

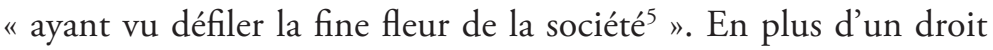
d'accès à l'université, c'est au sein de ces maisons tenues par le clergé catholique que l'élite canadienne-française a longtemps fait la conquête de la culture humaniste, composante essentielle du capital culturel des classes dominantes. Les traits caractéristiques associés aux collèges d'autrefois sont bien connus. Bastions des valeurs catholiques et des traditions canadiennes-françaises, défenseurs d'une culture générale très littéraire plongeant ses racines dans la Grèce et la Rome antiques, ils auraient résisté avec conviction aux sirènes du présentisme et aux impératifs techniques et matérialistes de tous ordres. On y formait, selon le mot de Montaigne, des têtes bien faites plutôt que des têtes bien pleines.

Pas si classiques que ça, les vieux collèges?

L'éclairage de l'historiographie récente

Malgré ses allures immémoriales, ce portrait des collèges et petits séminaires diocésains d'autrefois colle davantage à la réalité historique des décennies 1940, 1950 et 1960 qu’à celle des époques antérieures. Des études récentes montrent, en effet, le caractère en partie construit d'une telle image au moins jusqu'à la Seconde Guerre mondiale.

5 Denyse Baillargeon, "Les collèges classiques : nouveaux regards sur une vieille institution ", Historical Studies in Education = Revue d'histoire de l'éducation, vol. 21, no 1 (printemps 2009), p. 1, [En ligne], [http://historicalstudiesineducation. ca/index.php/edu_hse-rhe/issue/view/217/showToc]. Longtemps, le cours classique fut nécessaire pour accéder aux principales facultés universitaires : droit, théologie, médecine. La situation s'assouplira dans l'après-guerre. 
Ainsi, notre réflexe de penser le collège québécois comme une institution figée reproduisant à l'identique, depuis le premier établissement jésuite de 1635 jusqu’à la fin des années 1960, la tradition d'un Ratio studiorum conçu à la Renaissance, serait à remettre en cause.

S'intéressant à la trajectoire de l'enseignement humaniste, Paul Inchauspé rappelait à juste titre le contexte de fondation du réseau des collèges québécois : "Les premiers collèges étaient plutôt des collèges professionnels ", soulignait-il, " $[s]$ ur les douze collèges classiques créés entre 1840 et 1875 , huit étaient auparavant des collèges industriels commerciaux et agricoles ${ }^{6} "$. Les travaux de Christine Hudon sur le collège de Sainte-Anne-de-La-Pocatière étayent de manière plus précise cette idée. L'historienne soutient que l'institution du Bas-Saint-Laurent s'était particulièrement bien adaptée aux besoins de formation de la population rurale. Pour mieux la desservir, les autorités du collège avaient développé, en plus des humanités classiques, une formation pratique arrimée à la société marchande. Dès 1842, par exemple, le collège met en place un cours commercial - appelé aussi « cours anglais » - qui sera donné pendant plus de cent ans et accueillera un nombre important d'élèves ${ }^{7}$. On trouve aussi, dans la récente monographie de l'abbé Jean Panneton sur le Séminaire Saint-Joseph, des matériaux abondants qui déconstruisent l'image d'étanchéité au monde et d'immobilisme des anciennes institutions collégiales. Les notables laïques à l'origine de la maison trifluvienne fondée en 1860 et qui deviendra par la suite un séminaire diocésain avaient l'ambition initiale de développer concurremment diverses formations : classique, industrielle, commerciale et agricole.

${ }^{6}$ Paul Inchauspé, « Un nouvel humanisme, socle du nouveau système d'éducation proposé ", Bulletin d'histoire politique, vol. 12, n 2 (hiver 2004), p. 68.

7 C'est en 1946 que le cours commercial s'éteint. Christine Hudon, "Quelques observations et réflexions sur les projets éducatifs des collèges québécois pour garçons à partir d'un exemple : Sainte-Anne de La Pocatière au $19^{\mathrm{e}}$ siècle ", Historical Studies in Education = Revue d'histoire de l'éducation, vol. 21, $\mathrm{n}^{\circ} 1$ (printemps 2009), p. 24-40, [En ligne], [http://historicalstudiesineducation.ca/index.php/ edu_hse-rhe/issue/view/217/showToc]. 
C'est ainsi que, dès 1871 , le collège amorce sa diversification en la justifiant de cette façon : trop d'élèves quittent le cours classique après deux ou trois ans sans leur baccalauréat en poche, mais en ayant consacré beaucoup - trop - de temps à l'étude du grec et du latin ${ }^{8}$. À Trois-Rivières désormais, selon un modèle que partageront d'ailleurs plusieurs établissements au Québec, les premières années de scolarisation sont consacrées au cours commercial; seuls les élèves poursuivant au-delà du troisième ou quatrième degré s'adonnent aux humanités gréco-latines. On voit là s'exprimer le sens pratique de dirigeants qui, loin de miser uniquement sur la formation d'une élite universitaire et sacerdotale, s'engagent à fournir des outils de développement à une plus large portion de la jeunesse.

On pourrait penser que cette plasticité des maisons d'enseignement secondaire fut le fait exclusif de milieux ruraux composant avec la pauvreté et la rareté des ressources éducatives au XIX ${ }^{e}$ siècle. Mais, là encore, il convient de nuancer cette impression à la lumière de travaux récents sur la réalité urbaine. Retraçant le parcours des élèves du collège de Montréal à partir des registres d'inscription et de compte, Ollivier Hubert observe, pour sa part, la grande hétérogénéité des groupes de garçons (différences d'âge, d'origine géographique, de statut - pensionnaire ou externe) qui fréquentent l'institution. Ces élèves suivent, de surcroît, des parcours scolaires diversifiés (école anglaise, école française) et à durée variable. Le spectre des acquis éducatifs se révèle donc fort étendu, estime l'auteur, allant d'une littéracie de base à l'approfondissement de la philosophie. Il ne faut pas perdre de vue, toutefois, qu'une minorité seulement des élèves (environ $10 \%$ ) termine le cours classique au cours des années 1789 à 1860. Vraiment, la marche sera longue avant que ce collège ne s'impose comme le bastion élitaire auquel on a tendance à l'associer aujourd'hui'.

${ }^{8}$ Jean Panneton, Le Séminaire Saint-Joseph de Trois-Rivières : 1860-2010, Québec, Éditions du Septentrion, 2010.

9 Ollivier Hubert, «De la diversité des parcours et des formations dans les collèges du Bas-Canada : le cas de Montréal (1789-1860) ", Historical Studies in 
Soulignons enfin que la réalité plurielle des collèges que nous venons d'évoquer n'est pas propre aux institutions naissantes du $\mathrm{XIX}^{\mathrm{e}}$ siècle. C'est du moins ce que montre l'Histoire du Séminaire de Mont-Laurier, de Félix Bouvier, qui met en lumière le rôle significatif de l'institution dans le développement "intégral " de la région des Hautes-Laurentides au cours de la période 1910-1965. Si ce séminaire diocésain a contribué à la formation de l'élite cléricale et professionnelle, il a aussi investi de manière soutenue dans la préparation des classes moyennes grâce à une offre de formation diversifiée. En plus des cours commercial et classique, souligne l'auteur, l'institution laurentienne a mis en place une école d'arts et métiers ainsi qu'une école d'agriculture ${ }^{10}$.

Comme nous avons antérieurement tenté de l'exposer ${ }^{11}$, il faut attendre les années 1920 et les efforts des communautés de frères enseignants en vue de développer un niveau secondaire - le primaire supérieur - à vocation commerciale et technique, pour que le collège québécois se "classicise » véritablement. Dans un effort progressif de se distinguer des formations concurrentes, les collèges seront, en effet, contraints de préciser leur vocation en abandonnant les filières d'enseignement pratique qu'ils avaient mises sur pied antérieurement. L'après-guerre verra triompher ce collège classique et clériconationaliste que la mémoire retiendra comme pérenne; l'image d'Épinal sera enfin fixée.

Education = Revue d'histoire de l'éducation, vol. 21, no 1 (printemps 2009), p. 19-43, [En ligne], [http://historicalstudiesineducation.ca/index.php/edu_hse-rhe/issue/ view/217/showToc].

${ }^{10}$ Félix Bouvier, Histoire du Séminaire de Mont-Laurier : formation d'une élite et d'une classe moyenne, Montréal, Éditions Fides, 2005.

11 Louise Bienvenue, "Former à l'académie commerciale ou au collège classique? Un débat sur l'enseignement secondaire des garçons au début des années 1920 ", Historical Studies in Education = Revue d'histoire de l'éducation, vol. $21, \mathrm{n}^{\circ} 1$ (printemps 2009), p. 4-23, [En ligne], [http://historicalstudiesineducation.ca/ index.php/edu_hse-rhe/issue/view/217/showToc]. 


\section{Les collèges d'après-guerre : des institutions puissantes mais sujettes à la critique}

L'après-guerre marque-t-il l'âge d'or du cours classique? C'est l'époque, en tout cas, où le réseau d'institutions offrant cette formation atteint sa plus forte expansion. La période est marquée par une croissance économique et démographique significative, et cette prospérité amène les familles à investir davantage dans l'éducation de leurs enfants. Or ce nouvel enthousiasme pour l'éducation crée une pression sur le système; la demande est forte et l'offre, résolument insuffisante. Se posent déjà avec acuité de multiples questions concernant le financement et la nécessaire coordination du système scolaire dont témoigne l'intense débat public autour de la question qui se prolonge tout au cours des années 1950 et préfigure les délibérations de la commission Parent. L'exemple habituellement employé pour témoigner de cette fébrilité est la commission Tremblay, qui portait sur les problèmes constitutionnels, mais où 140 des 250 mémoires déposés abordaient des questions touchant l'éducation ${ }^{12}$.

Dans ce contexte particulier, la charge critique à l'endroit des collèges privés, monopole du clergé séculier et régulier, est intense, elle aussi. Peut-être n'a-t-on autant parlé du cours classique qu'en ce mitan du $\mathrm{XX}^{\mathrm{e}}$ siècle où chacun inventorie minutieusement ses vices et ses vertus. Bien sûr, ce n'est pas d'hier que se déploie la critique des collèges ${ }^{13}$, mais force est de constater que la fronde s'amplifie.

12 Claude Lessard, "Arthur Tremblay : contribution à l'étude des problèmes et des besoins de l'enseignement dans la province de Québec », dans Claude Corbo (dir.), Monuments intellectuels québécois $d u X X^{e}$ siècle : grands livres d'érudition, de science et de sagesse, Québec, Éditions du Septentrion, 2006, p. 128. Voir aussi Andrée Dufour, "Les années 1950 : une décennie annonciatrice de grands changements ", Bulletin d'histoire politique, vol. 12, n 2 (hiver 2004) p. 21.

13 Robert Gagnon, "Capital culturel et identité sociale : les fonctions sociales du discours sur l'encombrement des professions libérales au XIX ${ }^{\mathrm{e}}$ siècle ", Sociologie et sociétés, vol. 21, no 2 (automne 1989), p. 129-146, [En ligne], [http://www.erudit. org/revue/socsoc/1989/v21/n2/001268ar.pdf]. Voir aussi Louis Fréchette, Lettres à l'abbé Baillargé : à propos d'éducation, Montréal, Bibliothèque québécoise, 2003. 
Dans le journal Le Jour, par exemple, le journaliste Jean-Charles Harvey réclame des changements en profondeur, dont un cours secondaire plus accessible inspiré du modèle du high school, qui offrirait des spécialités diverses comme les sciences - ces mal-aimées des collèges classiques - et le commerce ${ }^{14}$. Mais sans surprise, c'est le monopole du clergé sur l'éducation préuniversitaire que l'hebdomadaire libéral fustige avant tout : «Aussi longtemps que l'Instruction publique, à tous les degrés, ne sera pas entre les mains de l'État, comme autorité suprême, la province de Québec ne pourra se vanter de vivre sous un régime démocratique ${ }^{15}$. "Des intervenants plus modérés, tel Ignace Brouillet, président de l'Association canadiennefrançaise pour l'avancement des sciences (ACFAS), appellent également de leurs vœux des réformes conséquentes, " après tout nous ne vivons pas dans une civilisation de mandarins et nous devons armer solidement nos enfants pour les combats qui les attendent ", affirme ce dernier en $1950^{16}$.

Par ailleurs, au cours des mêmes années, le développement de " sections classiques" au sein des commissions scolaires publiques ${ }^{17}$ n'est pas sans inquiéter les autorités des "vrais " collèges et petits séminaires diocésains qui craignent la concurrence d'un secteur public gratuit $^{18}$. Pour ces raisons, entre autres, la Fédération des collèges

${ }_{14}$ Paul Riverin [pseud.], "Baptiste aura ses high schools », Le Jour, vol. 9, n 24 (16 février 1946), p. 8 ; voir aussi Jean-Charles Harvey, " Monopole colossal ", Le Jour, vol. 9, n 10 (10 novembre 1945), p. 1, cités dans Johanne Rochette, Les débats sur l'enseignement des sciences dans les collèges classiques au Québec, 1929-1953, mémoire de maîtrise (histoire), Montréal, Université de Montréal, 1991, p. 262.

15 Paul Riverin [pseud.], "Collège et université », cité dans Rochette, Les débats sur l'enseignement, p. 263.

16 Ignace Brouillet, «Discours présidentiel : où en sommes-nous dans l'enseignement pré-universitaire? ", Annales de l'ACFAS, vol. 18 (1952), p. 51, cité dans Rochette, Les débats sur l'enseignement, p. 306.

17 Claude Galarneau compte 58 « sections classiques » intégrées au réseau public au cours de la période 1940-1969 (Les collèges classiques au Canada français, p. 66).

18 L'expansion du secteur public est certes considérable car, à la fin des années 1950, $45 \%$ des étudiants de l'Université de Montréal viennent déjà de l'enseignement secondaire public (Jean Gould et Nicole Gagnon, "De l'école à l'université : 
classiques est créée en 1953. Un front commun semble devenu nécessaire pour défendre les intérêts d'institutions naguère encore si jalouses de leur autonomie ${ }^{19}$.

Malgré ces développements et la grogne qui sourd de segments de plus en plus larges de la population, peu de gens parieraient au cours des décennies 1940 et 1950 sur la fin imminente du cours classique. Des voix autorisées, comme celle du futur sous-ministre de l'Éducation Arthur Tremblay, considèrent le scénario parfaitement invraisemblable. Le problème des collèges, estime ce dernier, n'est pas tant la concurrence du système public que la gestion de leur propre croissance ${ }^{20}$. Le développement des collèges est, en effet, phénoménal, comme l'a établi Claude Galarneau : alors que de « 1635 à 1939, 68 collèges avaient été fondés sur l'ensemble du territoire québécois », « [l]a période 1940-1965 voit apparâtre à elle seule 135 institutions privées $[\ldots]^{21}$ ».

quelle scolarisation? ", dans Fernand Dumont (dir.), La société québécoise après 30 ans de changements, Québec, Éditions de l'IQRC, 1990, p. 132.

19 Le climat d'incertitude semble agir comme un aiguillon sur les collèges, les entraînant dans un effort inégalé de modernisation administrative et pédagogique dans les années 1960 : réforme du programme, aménagement des immeubles pour le sport et l'intimité, introduction des méthodes actives dans l'enseignement, disparition du grec (Gould et Gagnon, «De l'école à l'université », p. 131-141). Voir également le film documentaire de Pierre Patry, Collège contemporain, Office national du film du Canada, $20 \mathrm{~min}, 1960$.

${ }^{20}$ Il est alors professeur à l'École de pédagogie et d'orientation de l'Université Laval. Son étude est financée à hauteur de 100000 \$ par la Fondation Carnegie pour procéder à une enquête rigoureuse sur l'état de l'éducation au Québec. Il sera plus tard membre adjoint sans droit de vote à la commission Parent puis, au sein du jeune ministère de l'Éducation, il occupera le poste de sous-ministre (Lessard, "Arthur Tremblay ", p. 134).

21 Si l'ensemble des régions géographiques profite de cette croissance, il faut préciser, cependant, que les institutions n'offrent pas toutes le cours complet de huit ans et que la majorité se spécialise dans le premier cycle dans un esprit d'accessibilité. Un certain nombre de ces institutions auront aussi la vie brève (Galarneau, Les collèges classiques au Canada français, p. 66). 


\section{Les anciens collèges : des traces de pierres et de mots}

Mais après le triomphe vint la chute. Le rapport Parent en appelle à un nouvel humanisme contemporain ${ }^{22}$, et le gouvernement Lesage décide que celui-ci ne s'enseignera pas dans les collèges, mais bien au sein de nouvelles créations que sont les polyvalentes et les cégeps. Depuis, le collège classique survit sous forme de traces. Les anciennes institutions, reconverties pour plusieurs en écoles secondaires privées, cultivent à leur façon la mémoire du vénérable cours classique : les anniversaires, par exemple, sont autant d'occasions d'en raviver le souvenir. Plusieurs maisons profitent de ces anniversaires pour organiser des événements et faire paraître des ouvrages commémoratifs. Ainsi, on a souligné récemment le $150^{\mathrm{e}}$ anniversaire du Séminaire Saint-Joseph de Trois-Rivières de même que le $200^{\mathrm{e}}$ anniversaire du Séminaire de Saint-Hyacinthe ${ }^{23}$, qui ont donné lieu à des publications dans lesquelles est évoqué un ancien temps où se mêlent, dans une sorte de folklore, les redingotes rigides, les coups pendables du pensionnat, les concours de rhétorique, les leçons de grec et la gastronomie approximative des réfectoires. Les grandes figures qui font la gloire de ces maisons - hommes politiques, évêques, artistes - ont aussi droit à leurs pages réservées. Les moments forts ponctuant la vie des anciennes communautés collégiales sont également relatés : tournois sportifs, joutes oratoires, concours d'académies littéraires, retraites de vocation et cérémonies des rubans figurent comme autant d'épisodes structurants dans la vie de jeunes hommes en formation. Ces récits et éphémérides regroupés font écho à ce que l'on retrouve de façon plus dispersée dans les mémoires et autobiographies d'anciens collégiens, comme ceux patiemment

22 Claude Corbo, "L'éducation et le nouvel humanisme contemporain ", L'éducation pour tous : une anthologie du Rapport Parent, Montréal, Les Presses de l'Université de Montréal, 2002, p. 65-80.

23 Diane Leblanc, Le Séminaire de Saint-Hyacinthe : deux siècles d'histoire, 200 ans d'éducation (1811-2011), Montmagny, Les Éditions Marquis, 2011; Panneton, Le Séminaire Saint-Joseph de Trois-Rivières, 1860-2010. 
colligés par Claude Corbo ${ }^{24}$. De plus en plus, semble-t-il, les ouvrages maison commandités par les collèges mettent aussi en évidence les trésors patrimoniaux que renferment les vieux murs de pierres grises: livres rares, tableaux de valeur, artéfacts des anciens musées des sciences naturelles, reliques religieuses rapportées de Rome ou de Jérusalem par quelques prêtres. Traces matérielles de ce que le Canada français avait de Beau et de Grand, les collèges incarnent un patrimoine qui gagne en noblesse, mais aussi en exotisme, à mesure que le temps passe. Par ailleurs, les machines bien huilées que sont les associations d'anciens, les amicales et conventums font le reste pour perpétuer la mémoire des collèges, qui peut encore prendre assise sur la solidarité des camarades. Huit ans collégiens pensionnaires ${ }^{25}$, comme l'affirme le titre d'un ouvrage souvenir préparé par des élèves du $122^{\circ}$ cours du collège de Sainte-Anne-de-La-Pocatière, ça crée des liens!

Mais pour plusieurs personnes, moins directement touchées par l'institution et son histoire, le collège classique est avant tout une mémoire de pierres. Il faut dire que plusieurs de ces établissements figurent de belle manière dans nos villes et villages. Ces élégants bâtiments, avec colonnes ou pilastres, pignons, lucarnes et portails, en imposent; ils sont un peu - avec les églises et autres ensembles conventuels - comme nos châteaux à nous ${ }^{26}$. De " magnifiques collèges ", des " citadelles nationales ", c'est ainsi qu'Étienne Parent en parlait en son temps ${ }^{27}$. $S^{\prime}$ il ne référait pas prioritairement à leurs qualités esthétiques, il reste que plusieurs de ces immeubles sont désormais inscrits au Répertoire du patrimoine culturel du Québec

${ }^{24}$ Claude Corbo, La mémoire du cours classique, Montréal, Éditions Logiques, 2000.

25 Paul Mathieu et Jean-Louis Levesque (dir.), Huit ans collégiens pensionnaires, Sherbrooke, Éditions de l'UTA, 2002. L'ouvrage a été préparé à l'occasion du $175^{\mathrm{C}}$ anniversaire de la maison.

${ }^{26}$ François Roy, "Au temps des collèges classiques : voyage dans le temps ", Le Nouvelliste, 28 septembre 2002, p. 36.

27 Cité dans Lionel Groulx, Professionnels et culture classique, causerie prononcée au Séminaire de Sainte-Thérèse à la réunion annuelle des Anciens, le deux mai mil neuf cent quarante-huit, [s. l., s. n.], 1948, p. 3. 
et y font l'objet de classements divers ${ }^{28}$. Dans un Québec qui a tant sacrifié son patrimoine bâti au profit de promoteurs cupides, on peut se réjouir de ce que plusieurs de ces édifices aient échappé au pic des démolisseurs en devenant soit des écoles secondaires privées, soit des cégeps ou les deux. Au gré de restaurations nécessaires, la mise en valeur de ce patrimoine architectural a parfois constitué une pièce maîtresse de la revitalisation des centres-villes. L'exemple du boulevard L'Ange-Gardien de l'Assomption est probant. Sur cette artère où sont désormais installés cafés et restaurants branchés ainsi que le théâtre Hector-Charland, le collège fondé par Jean-Baptiste Meilleur en 1832 figure comme un joyau. Il bénéficie, le soir, d'un éclairage étudié qui ne manque pas de faire son effet.

Le collège classique et la figure du collégien pensionnaire d'autrefois survivent aussi, autrement, à travers certaines œuvres de fiction. On en trouve une évocation fugitive dans Les belles-sours, de Michel Tremblay $^{29}$. La pièce de théâtre Les Feluettes de même que son adaptation cinématographique situent, pour leur part, certains passages de l'action dramatique dans un vieux collège. Sous la plume du dramaturge Michel Marc Bouchard, c'est la culture du théâtre classique et les relents homosensuels de ces milieux clos qui sont rappelés à la mémoire ainsi que l'intolérance paradoxale d'une époque vis-à-vis des amitiés particulières ${ }^{30}$. Par ailleurs, du point de vue d'une certaine identité masculine québécoise que les collèges ont contribué à façonner, les figures contrastées des frères de la famille Plouffe sont aussi bien inscrites dans nos imaginaires. Magnifiquement réincarnées dans le film de Gilles Carle, au tournant des années 1980, elles évoquent en concentré la cohabitation parfois difficile de la virilité avec la culture savante. Dans le coin droit, Guillaume, l'athlète,

${ }^{28}$ Ministère de la Culture et des Communications du Québec, "Répertoire du patrimoine culturel du Québec ", [En ligne], [http://www.patrimoine-culturel. gouv.qc.ca/rpcq] (26 juin 2013).

29 Michel Tremblay, Les belles-sœurs, Montréal, Leméac éditeur; Arles (France), Actes Sud, [1968] 2007.

${ }^{30}$ Michel Marc Bouchard, Les feluettes ou La répétition d'un drame romantique, $2^{\mathrm{e}}$ éd., Montréal, Leméac éditeur, 1988. 
champion de fers et de baseball, nourri aux comics books. Il rêve de Cincinnati tout en ne craignant pas, dans l'intervalle, d'affronter à vélo les rues pentues du Vieux-Québec. C'est aussi un séducteur. Dans le coin gauche, Ovide, amateur de beaux-arts, d'opéra et de grande littérature, est tenté par l'idée d'une vocation qui serait peutêtre surtout une échappatoire. Parlant une langue châtiée qui ne trouve pas son public, il est isolé par une culture classique qui se révèle un obstacle à la communication. Dans les milieux modestes, les acquis livresques s'inscrivent en faux contre la doxa masculine populaire, qui mise plutôt sur la force physique, le sens pratique et la maîtrise de l'anglais. On retrouve là les fameuses « tapettes du classique ", auxquelles fait référence Lucien Bouchard dans son autobiographie ${ }^{31}$. Cela revient à dire que la culture, si belle et si grande soit-elle, est toujours potentiellement un péril pour le jeune homme qui l'acquiert. N'est-ce pas un peu ce qu'exprime la tirade devenue classique : "Y'a pas de place nulle part pour les Ovide Plouffe du monde entier ${ }^{32}$ "?

À la recherche des traces vivantes du cours classique, on se tourne aussi, bien sûr, vers la classe politique. Malgré le renouvellement des générations et l'entrée significative d'hommes et de femmes formés dans les polyvalentes, les écoles privées et les cégeps, une partie des parlementaires actuels - pour ne parler que de cette fraction du groupe dirigeant - et nombre d'anciens politiciens toujours présents sur la scène publique portent encore l'empreinte du cours classique. Rappelons que de Pierre-Joseph-Olivier Chauveau jusqu’à Jean Charest $^{33}$ qui mettait fin, pensions-nous, à cette longue tradition, presque tous les premiers ministres du Québec ont fait leurs études classiques $^{34}$. En 1998, un éditorialiste du Quotidien de Chicoutimi

31 Lucien Bouchard, À visage découvert, Montréal, Éditions du Boréal, 1992, p. 31.

32 Gilles Carle, Les Plouffe, production Justine Héroux, 1981. Le film est inspiré du roman de Roger Lemelin (Québec, Bélisle, 1948).

33 Jean Charest a fait son cours secondaire à la polyvalente Montcalm de Sherbrooke.

34 Selon le site de l'Assemblée nationale du Québec, il y aurait cinq exceptions à la règle : Henri-Gustave Joly de Lotbinière (Paris), Joseph-Alfred Mousseau (Académie de Berthier), Simon-Napoléon Parent (école normale), Antonio Barrette 
décrivait d'ailleurs l'affrontement de Jean Charest et de Lucien Bouchard sur la scène politique québécoise comme celui du " cours classique contre les cégeps ${ }^{35}$ ». Or si l'on pensait alors que Charest avait mis un terme à une trame quasi ininterrompue, c'était avant que Pauline Marois, en digne diplômée du collège Jésus-Marie de Sillery où elle a fait son cours classique, reprenne le fil rompu, mais dans une version féminine.

Une telle formation a-t-elle laissé une marque particulière, une couleur précise, sur notre culture politique québécoise? La question est importante et mériterait une étude plus approfondie que celle que nous développons ici. On pense immédiatement, néanmoins, à l'enseignement de la rhétorique, cet art mis en exergue par les collèges et qui préparait entre autres, rappelons-le, à la prédication religieuse. Vient aussi à l'esprit l'influence probable d'une approche éducative généraliste, destinée à former le jugement, à développer le goût du bien commun, à conserver en toute chose un sens clair des finalités. Certes, des modèles viennent en tête : un Lucien Bouchard, élève du collège de Jonquière, citant Cicéron et séduisant les foules par sa harangue emportée. Un Bernard Landry multipliant les locutions latines avec une diction soignée qui n'est sans doute pas étrangère au Séminaire de Joliette qu'il a fréquenté ${ }^{36}$. Mais il faut se garder de trop de généralités à ce chapitre. S'il y a un habitus hérité de la formation collégiale, un style bien " cours classique " comme on se plaît à le dire, il est difficile de conclure à sa transposition dans une orientation

(Académie Saint-Viateur), Daniel Johnson (fils) (lieu de scolarisation secondaire inconnu), Jacques Parizeau (collège Stanislas). [En ligne], [http://www.assnat. qc.ca/fr/ patrimoine/pm.html] (26 juin 2013).

35 Carol Néron, "Bouchard-Charest : le cours classique contre les cégeps ", Le Quotidien, 31 mars 1998, p. 8. L'auteur dit emprunter cette idée à un reporter de la tribune de l'Assemblée nationale qu'il ne nomme pas.

36 Dans son film documentaire $\grave{A}$ hauteur d'homme (Productions Virage, 2003), Jean-Claude Labrecque fait bien ressortir ces traits, entre autres, dans une scène où Bernard Landry répète plusieurs fois la même locution latine - Audi alteram partem - lors d'une conférence de presse. 
et un style d'action particuliers. Car chez les finissants du cours classique, n'y a-t-il pas autant d'orateurs de talent que de rhétoriciens malhabiles, d'indépendantistes convaincus que de fiers fédéralistes, de leaders aux sensibilités sociales assumées que d'amis du laisser-faire, de personnages aux allures populistes que de chefs à la prestance plus distinguée, d'hommes d'État ayant un sens aigu de l'histoire que de carriéristes soucieux de la prochaine élection?

Il y a, au demeurant, dans l'héritage des anciens des collèges et séminaires tout un rapport à la culture catholique, qui reste à approfondir, mais qui a assurément moulé les consciences et le sens moral de nos dirigeants, quelle que soit la façon dont a évolué, par la suite, leur rapport au religieux. Sait-on qu'au moins deux de nos anciens premiers ministres récents, Daniel Johnson père (qui a fréquenté le Séminaire de Saint-Hyacinthe) et Lucien Bouchard, ont raconté avoir été tiraillés par le sacerdoce, cette forme supérieure d'engagement si valorisée par les prêtres enseignants ${ }^{37}$ ?

Certes, l'histoire du collège classique nous instruit surtout sur la formation de l'élite masculine, mais la chose n'est pas exclusive. Par exemple, au lendemain du décès de la mairesse Andrée Boucher de Québec, une ancienne consœur de classe lui rendait hommage dans les pages du Soleil de Québec, rappelant comment sa formation classique au collège de Sillery dans les années 1950 avait moulé la jeune oratrice :

Andrée avait sûrement commencé à exercer son verbe très jeune; mais à mesure que nos études avançaient, son imaginaire nourri par le récit de ces épopées, son vocabulaire enrichi par ses études, avec le sens de l'humour qu'on lui connait, elle s'amusait à lancer des

37 Lors de la cérémonie du ruban qui suivait la retraite de fin d'études, le moment culminant était l'annonce des vocations religieuses, auxquelles on réservait le ruban blanc. On peut entendre les propos de Lucien Bouchard à l'émission J'avais 20 ans de Radio-Canada, 17 février 2007, [En ligne], [http:/www.radio-canada.ca/radio/ profondeur/20ans.html]. Voir aussi Pierre Godin, Daniel Johnson, t. 1 : 1946-1964, la passion du pouvoir, Montréal, Éditions de l'Homme, 1980. 
débats durant les récrés, empruntant le langage d'un Charlemagne, jouant le rôle d'un César, Hannibal ou Scipion l'Africain. [...] Tout sujet de débat était traité à la romaine, à la grecque et prenait un ton de guerre ${ }^{38}$.

\section{Le débat pédagogique des vingt dernières années}

Là où le collège survit avec une étonnante vitalité, comme référence positive mais aussi parfois négative, c'est au sein du débat pédagogique. À partir d'articles de la presse écrite francophone ${ }^{39}$ et d'essais portant sur l'éducation publiés au cours des vingt dernières années, nous allons tenter de faire saillir la référence au cours classique pour en montrer la variété et la durabilité des usages ${ }^{40}$. Nous examinerons d'abord deux thèmes pour lesquels la mémoire du classique est invoquée fréquemment : la transmission de la culture et la qualité de la langue. Nous présenterons ensuite d'autres types d'intervention autour de questions scolaires qui font également appel au modèle de l'ancienne institution.

\section{La transmission de la culture}

Le thème de la transmission de la culture semble particulièrement propice à l'évocation du cours classique. Au tournant des années 1990, Nicole Gagnon et Jean Gould trempaient leur plume dans le vitriol pour analyser l'évolution de la scolarisation des Québécois depuis les grandes réformes de la Révolution tranquille. Le jugement posé par les auteurs était sans appel : tout est à jeter aux orties. En plus de s'être technocratisé, le secondaire démocratique - « c'est-à-

38 Claire Vézina, "Souvenir de collégiennes avec Andrée ", Le Soleil, 2 septembre 2007, p. 23.

39 Le repérage d'articles de presse a été fait à partir de la base de données Eureka.cc. La recherche a été menée à partir de mots clés, tels " humanités ", " collèges classiques ", " cours classiques ", pour les années 1990 à 2013.

${ }^{40}$ La démarche sera forcément exploratoire; le discours sur l'éducation étant un continent, tenter d'en rendre compte d'une manière systématique est un défi; nous avons tenté d'en faire un bon tour d'horizon en sachant que des pièces pourraient éventuellement s'ajouter au dossier. 
dire, calibré sur la moyenne inférieure " - se serait également déshumanisé. « Personne n'habite plus l'école ni le savoir. La vie se passe ailleurs ", se plaignaient les sociologues dans une argumentation où la référence aux anciennes humanités était persistante :

On a démoli des institutions d'éducation pour fabriquer un système de scolarisation destiné à répondre aux besoins de la société ou de l'individu. L'école est devenue une grande gare de triage des aptitudes vers les divers secteurs du marché du travail. Le savoir a été oublié au profit de la mise en valeur de la ressource humaine. Au lieu de transmettre la culture, c'est-à-dire d'y introduire la jeune génération, on prétend répondre à ses besoins, qui sont en réalité les besoins de l'économie en main-d'œuvre informée. Dès la petite école, on prend prétexte de la spontanéité des enfants pour leur cultiver ladite " créativité " qui est au fond une première forme de comportement productif. Les adolescents se font corner les oreilles par des cours de choix de carrière qu'ils sont les premiers à trouver " complètement débiles » et se font initier aux mystères de la Bourse, alors qu'ils seraient tout disposés à apprendre le latin ${ }^{41}$.

Plus tard, au début des années 2000, dans le cadre de l'introduction controversée de la fameuse "réforme scolaire ", le modèle du cours classique s'immisçait à nouveau en filigrane des échanges. Dans ce contexte agité, le collectif de réflexion Le Pont entre les générations signait une lettre au Devoir sous le titre « Les sciences de l'éducation forment-elles de véritables maîtres? ». On y attirait l'attention du lecteur sur l'achoppement de la transmission des valeurs humanistes :

Voilà pourquoi l'école secondaire a un rôle si important. Elle n'est pas le seul lieu où on transmet l'héritage humaniste, mais c'en est certainement l'un des principaux. Elle doit certes fournir des savoirs pratiques, des habiletés techniques et une bonne préparation au marché du travail. Mais son autre grande finalité est de transmettre les connaissances de base, les valeurs de la société et du pays, le patrimoine culturel d'une civilisation.

${ }^{41}$ Gould et Gagnon, « De l'école à l'université », p. 135. 
Ce préambule établi, l'organisme amorçait une critique des " compétences transversales " destinées à prendre le pas, dans le contexte de la réforme, sur la culture elle-même. Un ressaisissement s'imposait, passant par un retour aux matières fondamentales comme le français et l'histoire, en conformité d'ailleurs avec les conclusions des États généraux sur l'éducation tenus quelques années auparavant. Or remettre ces matières au programme ne suffisait pas, il fallait aussi doter les écoles de maîtres compétents pour les enseigner. C'est là où le bât blesse, arguait Le Pont, puisque depuis 1994, les futurs enseignants sont tous impérativement inscrits en sciences de l'éducation plutôt que dans un programme disciplinaire et reçoivent, de ce fait, une formation moins consistante : «L'objectif est moins de transmettre des connaissances, un savoir, une culture, que de pouvoir "gérer une classe", "comprendre" la psychologie des enfants, saisir le "contexte de diversité" de l'école postmoderne ». Prévenant des attaques, Le Pont entre les générations se réclamait stratégiquement de la modernité du rapport Parent pour soutenir sa position : "Le recentrage de la formation des maîtres autour des matières de base n'a donc rien d'une nostalgie de l'ancien régime. Il constitue plutôt la réaffirmation de valeurs humanistes au cœur du projet de la Révolution tranquille ${ }^{42}$. »

Pourtant, quelques jours plus tard, toujours dans les pages du Devoir, le doyen de la Faculté d'éducation de l'Université du Québec à Montréal, Marc Turgeon, qualifiait les auteurs de la lettre d'« obsédés du paradis perdu ", faisant allusivement référence au modèle révolu du cours classique. "Ma mémoire personnelle de ce que fut l'enseignement secondaire est assez différente ", insistait le doyen, qui

${ }^{42}$ Le Pont entre les générations, "Les sciences de l'éducation forment-elles de véritables maîtres? ", Le Devoir, 23 janvier 2003 (à noter que l'auteure de cet article était membre du regroupement au moment de la parution des lettres). Pour une part stratégique, cet argument montre néanmoins que le débat est plus complexe qu'une simple opposition entre traditionalistes et modernistes. En effet, en regard de la radicalisation récente de la pensée pédagogique centrée sur l'élève (souvent présentée sous l'étiquette du socioconstructivisme), le rapport Parent apparaît désormais, aux yeux de plusieurs, comme une solution modérée par son souci de préserver le meilleur de la tradition humaniste tout en l'inscrivant dans la modernité. 
rappelait l'incurie pédagogique et psychologique de ses anciens maîtres : "Un cours de maths avec un prof me regardant d'un air hagard parce que je ne comprenais pas les rudiments de la trigonométrie et qui ne m'adressera plus la parole de l'année. "Turgeon s'en prenait aussi à la dimension foncièrement élitiste du cours classique : "La perte de la Tradition que le PEG déplore ne serait-elle que la nostalgie d'une reconnaissance publique des élites et de leur droit d'accéder à une éducation leur permettant de se reproduire ${ }^{43}$ ? »

Cet enjeu de la pérennité de l'héritage culturel, souvent conçu dans son acception nationale, est assurément un thème récurrent. On en trouve une nouvelle évocation en 2008, cette fois sous la plume du Collectif pour une éducation de qualité, dans un mémoire déposé à la commission Bouchard-Taylor :

Au Québec, la question de la transmission de la culture, comme héritage occidental ou comme patrimoine transmis par des générations de Québécois, est particulièrement sensible. La grande culture humaniste, que tentaient de transmettre, non sans défauts, les collèges classiques d'autrefois et dont il subsiste un reliquat dans les cégeps, est aujourd'hui frappée de suspicion. On la juge trop élitiste, pas assez pratique; elle aurait créé, chez les jeunes issus des milieux défavorisés, une forme malsaine d'inhibition. Cette culture humaniste des collèges classiques est également associée à un sombre passé, celui de la "grande noirceur " canadiennefrançaise, et à un clergé qui, selon une vulgate toujours en vogue, n'aurait cherché qu'à maintenir tout un peuple dans l'ignorance. Aujourd'hui, les écoles québécoises, bien qu'elles aient accompli de grands progrès dans la promotion de l'accès à l'éducation, font face à des pressions multiples, venant tant de l'État que du marché ou de la société civile, qui les soumettent à la valse-hésitation des changements de programme et d'approches pédagogiques où se perd la finalité de la transmission d'un patrimoine culturel.

43 Marc Turgeon, «Réplique au Pont entre les générations : les "obsédés du paradis perdu” n'ont pas raison ", Le Devoir, 29 janvier 2003, [En ligne], [http://www. ledevoir.com/non-classe/19233/replique-au-pont-entre-les-generations-lesobsedes-du-paradis-perdu-n-ont-pas-raison]. 
Si le cours classique sert à nouveau d'assise à une critique de l'école contemporaine et de l'ensemble des mécanismes de reproduction de la culture, le lien est plus clairement tissé dans ce mémoire entre la solidité de la transmission de l'héritage national et la capacité à accueillir sereinement l'immigration ${ }^{44}$. Dans le contexte d'audiences portant sur les pratiques d'accommodement reliées aux différences culturelles, l'association du collège classique à la Grande Noirceur n'est pas sans évoquer toute la difficulté qu'éprouvent encore les Québécois à métaboliser leur passé catholique et la fragilisation identitaire que représente parfois ce rapport douloureux au religieux.

Par ailleurs, au terme d'une décennie de mise en œuvre controversée d'une réforme scolaire axée sur la poursuite des " compétences ", il n'est pas anodin qu'un portrait de la ministre de l'Éducation Michelle Courchesne, paru dans le magazine L'Actualité en 2009, ait été intitulé : "J'ai une certaine nostalgie du cours classique ". Cellelà même qui a été saluée pour avoir mis un holà aux innovations pédagogiques des fonctionnaires en voulant rétablir le bulletin chiffré et la possibilité de faire redoubler les élèves, reconnaissait en entrevue sa foi dans le livre : "Pour moi, la lecture est vraiment essentielle dans une vie. Je crois beaucoup à son pouvoir. Internet et la télé, ce n'est pas suffisant. Comment peut-on se développer sans lire? » L'article évoquait ensuite le vœu de la ministre pour que la littérature retrouve une place importante à l'école et citait ainsi sa confession : "J'ai une petite nostalgie du cours classique [...]. Sans vouloir

${ }^{44}$ Collectif pour une éducation de qualité, Transmettre adéquatement un patrimoine culturel et historique, mémoire présenté dans le cadre des audiences de la Commission de consultation sur les pratiques d'accommodement reliées aux différences culturelles. Le texte est reproduit avec une introduction d'Éric Bédard et Marc Chevrier, «La transmission du patrimoine historique en péril : le Collectif pour une éducation de qualité (CEQ) se prononce ", dans Bulletin d'histoire politique, vol. 16, n 2 (hiver 2008), [En ligne], [http://www.bulletinhistoirepolitique.org/le-bulletin/numerosprecedents/volume-16-numero-2/la-transmission-du-patrimoine-historique-enperil-le-collectif-pour-une-education-de-qualite-ceq-se-prononce] (26 juin 2013). 
retourner en arrière, je crois que cette formation recélait quelques bons aspects qui mériteraient d'être recyclés ${ }^{45}$."

Plus récemment, le débat sur le changement de nom du programme collégial Arts et lettres a été une nouvelle occasion d'invoquer l'ancienne formation classique et ses vertus. Dans Le Devoir du 14 juin 2013, le professeur de littérature Jean Larose, pourfendeur de longue date des apories de l'école contemporaine, revenait à la charge à l'occasion d'une initiative du ministre de l'Enseignement supérieur, de la Recherche, de la Science et de la Technologie pour actualiser l'intitulé du programme Arts et lettres au cégep. Pour l'essayiste, la décision de Pierre Duchesne de renommer le programme Culture et communication représentait plus qu'une banale modernisation de vocable; elle constituait rien de moins qu'une "rupture historique ". On s'employait ainsi à retirer au patrimoine littéraire la dernière petite place qu'il occupait encore dans la formation générale pour l'inféoder plus parfaitement au complexe médiatico-numérique et à sa culture addictive de divertissement. "Au jeune poète qui espère Regards et Jeux dans l'espace, on apprendra à gérer son profil en ligne, à planifier la mise en marché de son œuvre future avec sa production. » Les décideurs qui agissent de la sorte, déplore encore le professeur de littérature, sont manifestement les représentants d'une génération nouvelle :

Il fut un temps au Parti québécois où l'on estimait que dans l'identité nationale comptait non seulement Joseph-Armand Bombardier mais aussi Gaston Miron. Gilles Vigneault n'était pas un entrepreneur culturel pour René Lévesque, ses poèmes n'étaient pas des produits artistiques. Jacques Parizeau n'aurait pas douté que les Lettres favorisent " le développement intégral de la personne ». Bernard Landry n'aurait pas eu la simplicité de croire que Communication est plus rassembleur que Lettres.

45 Danielle Stanton, "J'ai une petite nostalgie du cours classique" : portrait de Michelle Courchesne ", L'Actualité, vol. 34, no 8 (15 mai 2009), p. 37. 
Évoquant ainsi une ancienne génération de politiciens - celle formée au cours classique, aurait-il pu facilement ajouter -, Larose en remet : « Les politiques qui furent élus en 76 avaient lu. Ils avaient encore la naïveté de trouver le français beau et précieux (en ce tempslà, un ministre pouvait s'exprimer sans faire deux fautes de français par phrase). » L'essayiste s'attache ensuite à recomposer la généalogie ayant rendu possible l'« erreur " du changement de dénomination en remontant au rapport Parent :

Il y a deux générations, pour corriger l'injustice qui réservait le patrimoine classique à l'élite, au lieu d'ouvrir celui-ci à tout le monde, on l'a enlevé à tout le monde. Plus tard, le PQ légiférant pour sauver le français, étrangement n'a pas fait le rapport avec sa langue mère, le latin. On a sauvé l'arbre et coupé ses racines.

S'en prenant au présentisme qui impose à l'école une culture définie par le marché, il s'offusque : voilà comment on déshérite une jeunesse « d'une part essentielle de la culture nationale au profit du marché des biens culturels $\mathrm{s}^{46} \%$.

\section{La qualité de la langue}

Les humanités classiques attachaient beaucoup d'importance à la maitrise de la langue et, dans la pédagogie des collèges, l'apprentissage des langues anciennes était la voie royale pour atteindre à cette compréhension fine, garante d'un usage adéquat. On ne se surprendra pas que dans les débats sur la langue française et sur son enseignement - pléthoriques au Québec - la référence au cours classique surgisse à nouveau. À titre d'exemple, en 1999, dans un rapport du Conseil de la langue française, signé par le linguiste Jacques Maurais et intitulé La qualité de la langue : un projet de société, l'auteur notait les progrès importants accomplis depuis l'adoption de la Charte de la langue française en 1977. Or si l'objectif de la loi 101 était de faire

46 Jean Larose, "À l'école du présent ", Le Devoir, 14 juin 2013, [En ligne], [http://www.ledevoir.com/societe/education/380866/a-l-ecole-du-present]. 
du français la langue commune au Québec, force est de constater, affirmait en substance l'auteur, que présence n'égale pas qualité. Pour atteindre à cette qualité qui laisse toujours à désirer, les stratégies éducatives doivent être surveillées de près, martelait le rapport. Soutenant le principe selon lequel le colinguisme ou la comparaison entre deux langues est un excellent moyen d'améliorer la connaissance de la langue maternelle, Maurais rappelait avec insistance les qualités propres à l'enseignement classique, en ne se gênant pas pour écorcher au passage les pédagogies désormais à l'honneur, centrées sur l'expression de l'élève :

Au Québec, l'enseignement classique avait compris l'importance d'établir des comparaisons systématiques entre le français et les autres langues alors au programme (anglais, latin, grec). C'était, évidemment, avant l'âge d'or de la pédagogie du vécu et de l'oral [...]. Avec la disparition du grec et celle, presque complète, du latin de notre enseignement secondaire, avec l'arrivée de nouvelles méthodes dans l'enseignement de l'anglais, le travail de comparaison systématique entre deux systèmes linguistiques a été abandonné. Pourtant, le rapport Parent avait rappelé l'importance d'une réflexion de type comparatif sur la langue ${ }^{47}$.

On le constate, la valorisation de l'ancien cours classique est encore ici une façon de mettre en évidence, par effet de contraste, les faiblesses de l'école contemporaine. Débordant du strict sujet de la langue, l'auteur profite de l'exercice comparatiste pour faire passer son message concernant la pédagogie contemporaine, surtout orientée vers l'élève. Si l'expression de soi a la cote, semble observer Maurais, c'est au détriment d'un véritable apprentissage de la langue qui exigerait un plus grand décentrement.

Plus tard, en 2007, le thème de la maîtrise de la langue française est repris par Louis Cornellier, qu'on connaît surtout pour ses

47 Jacques Maurais, La qualité de la langue : un projet de société, rapport présenté au Conseil de la langue française, janvier 1999, p. 74, [En ligne], [www.cslf.gouv. qc.ca/publications/pubb147/b147.pdf] (22 juin 2013). 
chroniques au Devoir, mais qui s'exprimait, cette fois-là, dans l'hebdomadaire régional L'Expression. Sur ce sujet épineux, le journaliste formulait, pour sa part, un point de vue moins dramatique :

Avant la démocratisation de l'enseignement, très peu d'enfants québécois fréquentaient longuement le système scolaire. Les finissants du cours classique écrivaient peut-être à peu près sans faire de fautes, mais les autres, la majorité, abandonnaient l'école et, souvent, n'écrivaient plus.

Se référant à l'essai La grande aventure de la langue française, de Jean-Benoît Nadeau et Julie Barlow, Cornellier rappelait le très petit nombre de Québécois qui, il y a quelques décennies encore, pouvaient espérer terminer un cours classique. Tous les autres abandonnaient en chemin. Il faut donc applaudir au progrès que représente l'accès désormais massif à la scolarisation collégiale. Même si bon nombre de candidats qui en sortent écrivent mal, souligne le chroniqueur, au moins ils écrivent, et les meilleurs élèves d'aujourd'hui sont certainement aussi bons que les finissants du cours classique d'il y a un demi-siècle : "Toute la différence est là et ne pas y voir un progrès revient à ne voir que le mauvais côté des choses ${ }^{48}$. "

\section{Les autres thèmes}

Assurément, les thématiques qui soulèvent des enjeux identitaires sont les plus susceptibles de faire intervenir la référence aux vieux collèges du Canada français. Or le souvenir de ceux-ci ressurgit aussi à l'occasion d'autres propos. Par exemple, c'est sur un ton plus léger, celui de la douce nostalgie, qu'un collaborateur du Nouvelliste de Trois-Rivières abordait, en 2002, un thème moins fréquenté lorsqu'il est question de la bonne éducation, celui de la beauté des lieux et de la qualité d'un milieu de vie. Disant ne pas s'opposer à la modernité,

48 Louis Cornellier, "Qualité de la langue : pas de panique ", L'Expression, 21 novembre 2007, p. 6. 
le journaliste François Roy se souvenait néanmoins d'un autre temps, plus centré sur l'humain et la beauté :

Car, en fait, les collèges en question n'étaient pas seulement des «boîtes à cours ». Ils étaient des milieux de vie presque complets, où les arts et les sciences prenaient une place énorme. Quand on s'approchait d'un collège classique, le premier art que l'on remarquait, c'était bien sûr l'architecture [...]. Et puis, à l'intérieur des murs, on y retrouvait un théâtre, un musée, une chapelle pour le chant et la musique d'orgue, sans oublier un cabinet de sciences naturelles, avec toute une collection d'animaux empaillés.

La présence de la nature dans l'environnement collégial d'autrefois est ainsi rappelée par Roy, qui semble autant influencé par son imaginaire cinématographique que par l'histoire véridique des collèges québécois :

À l'extérieur, lorsque le collège trônait en milieu rural, on pouvait admirer une sorte de forêt aménagée, avec des sentiers sous les arbres, des étangs, de jolis ponts couverts, de même qu'une sorte d'enclos avec des bancs, pour les lectures publiques. Si vous avez vu le film «La Société des poètes disparus », vous savez sans doute que la poésie et la rhétorique se portent souvent mieux quand on les pratique au grand air $^{49}$.

Par ailleurs, la lancinante question du décrochage scolaire a également donné lieu à une comparaison entre l'ancien système et le nouveau. En 2003, la journaliste Marie-Andrée Chouinard du Devoir s'attaquait à ce problème alarmant en l'appréhendant dans sa dimension historique. Dans le cadre d'un dossier spécial sur les 40 ans du rapport Parent, elle interrogeait à ce propos Guy Rocher, personnage-clé de la célèbre Commission royale d'enquête sur l'enseignement dans la province de Québec (commission Parent) :

Bête noire de tout ministre de l'Éducation, le décrochage scolaire faisait aussi rage à l'époque du cours classique [...]. Ça frôlait

49 François Roy, "Au temps des collèges classiques : voyage dans le temps ", Le Nouvelliste, 28 septembre 2002, p. 36. 
parfois 70-75\%. On était une centaine en éléments latins et, à la fin, on n'était plus qu'une trentaine, parfois 25 . Le cours classique ne s'adaptait pas à nous, on s'adaptait au cours classique ${ }^{50}$ !

Sur ce terrain précis du décrochage, les nostalgiques du cours classique sont assurément moins diserts, sans doute parce que les chiffres sont accablants. À moins de revendiquer radicalement une école qui soit un lieu d'écrémage, on préfère laisser un voile pudique sur de telles statistiques. Les mêmes chiffres seraient aussi susceptibles de donner prise à ceux qui cherchent à interpréter les problèmes scolaires des garçons autrement que par l'argument d'une féminisation excessive de l'école ${ }^{51}$. Car à l'époque des collèges classiques de garçons, on ne pouvait reprocher au système d'être " trop féminisé ", considérant que s'y trouvait, essentiellement, un personnel enseignant masculin.

Enfin, la tourmente du « Printemps érable » de 2012 a entraîné un nouvel usage polémique du cours classique, une formation qui représentait pour plusieurs des déboursés considérables et proportionnellement plus importants que les actuels droits de scolarité universitaires en regard d'un revenu moyen. Dans une lettre ouverte au Devoir, par exemple, le professeur titulaire François Colbert, de HEC Montréal, appuyait l'intransigeance du gouvernement contre les associations étudiantes :

J'ai fait mes études universitaires entre 1969 et 1973. J'ai payé mes droits de scolarité, mon appartement, ma nourriture, mon habillement, et ensuite mes dettes d'études. J'ai aussi payé pour

50 Guy Rocher, cité dans Marie-Andrée Chouinard, «Les 40 ans du rapport Parent : le décrochage, du cours classique à aujourd'hui ", Le Devoir, 29 mars 2003, p. B6.

51 Pour un exemple de ce type de réflexion : "Les garçons ont besoin de bouger. L'école les tétanise, les ennuie. L'édu” est souvent leur matière préférée, où ils se défoulent et excellent. Les choix actuels des écoles de Jonquière [qui favorisent les arts] me semblent un symptôme de la féminisation étouffante de l'école " (Myriam Ségal, "La féminisation de l'école étouffe les garçons ", Le Quotidien, 31 mars 2009, [En ligne], [http://www.lapresse.ca/le-quotidien/opinions/chronique/200903/31/01841931-la-feminisation-de-lecole-etouffe-les-garcons.php]). Le recul historique invite certainement à ne pas simplifier de la sorte cette question complexe de l'échec et du décrochage des garçons. 
les droits de scolarité de mon cours classique, car mes parents n'avaient pas les moyens de payer. Si je l'ai fait au coût projeté pour les étudiants actuels dans cinq ans, eux aussi peuvent le faire! Mes amis d'alors qui étudiaient en histoire, en philosophie, en théâtre étaient dans la même situation que moi ; nous avons travaillé et nous avons réussi, nous ne sommes pas morts. Nous avons fait des sacrifices. Malheureusement, les mots "sacrifice " et « efforts " semblent avoir disparu du dictionnaire ${ }^{52}$ !

On le constate, l'époque du collège classique, c'est aussi, dans l'imaginaire de bien des protagonistes du débat pédagogique, l'époque révolue d'une culture de l'effort et de la persévérance que plusieurs souhaiteraient voir réhabilitée auprès de la jeunesse contemporaine.

\section{Conclusion : que reste-t-il du cours classique?}

Qu'il s'agisse de la formation des maîtres, de l'enseignement du français, des droits de scolarité ou du rapport à l'effort, la référence au cours classique semble demeurer encore bien vivante et efficace au sein de l'agora. Épouvantail pour les uns, joyau sacrifié à l'autel de la modernité pour les autres, on évoque sa mémoire, figée dans son glorieux modèle d'après-guerre, pour soutenir diverses argumentations concernant l'éducation. Il y a fort à parier qu'une enquête plus minutieuse encore, examinant les thèmes de l'enseignement de l'histoire, de la discipline à l'école ou des vertus de l'uniforme, enrichirait encore davantage ce bilan déjà substantiel des usages contemporains du cours classique. Or la référence n'est pas revendiquée également par tous, il est vrai. Si certains évoquent le collège classique comme un contre-modèle, représentant d'un temps moins démocratique et assurément plus sexiste, par exemple, ce sont surtout les contempteurs du modèle scolaire actuel et les hérauts d'un nationalisme québécois irrigué de sa source canadienne-française qui ont le plus souvent les mots " humanités " et " collèges classiques "

52 François Colbert, "J'appuie notre gouvernement! », Le Devoir, 21 mars 2012, p. A8. 
à la bouche puisqu'ils les ont, également, au cœur. Ce réflexe s'éteindra-t-il avec les derniers diplômés? Le Québec perdra-t-il un jour définitivement son latin? La mémoire vive risque certes de s'atténuer, mais il semble, étonnamment, que le collège soit toujours dans la mire de certains commentateurs qui n'étaient pas même nés au moment où les derniers établissements ont fermé leurs portes. Lisons, en guise de conclusion, Mathieu Bock-Côté dans une chronique du Journal de Montréal portant sur «La bonne éducation ", en février 2013 :

Pourquoi sentons-nous que l'école québécoise a fait faillite? Je ne parle pas d'une faillite financière, mais d'une faillite morale et culturelle. La réponse est simple : c'est parce qu'elle ne comprend plus sa propre mission. Elle ne sait plus ce que veut dire éduquer. [...] Cette révolution délirante a mené au désastre. L'homme n'est pas « libéré », il est déculturé, déraciné. L'essentiel n'est pas de pomper des milliards en éducation, mais d'imposer un changement de philosophie. Il faudra en revenir à une conception classique de l'éducation ${ }^{53}$.

Si une nouvelle génération de tribuns brandit ainsi le modèle de la formation classique, on peut penser que ce lieu de mémoire a encore quelques belles années devant lui. Certaines initiatives, comme la fondation récente du Collège néo-classique, qui entend «offrir le meilleur des études classiques aux leaders de la génération montante ${ }^{54}$ ", laissent penser que le modèle des humanités gréco-romaines risque d'agir encore durablement comme une sorte de contrepoids aux tendances présentistes, voire instrumentales de l'école contemporaine. Ad multos annos! Non, le collège classique n'est pas mort.

${ }^{53}$ Mathieu Bock-Côté, " La bonne éducation : autrefois, l'homme avait des devoirs envers le monde ", Le Journal de Montréal, 19 février 2013, [En ligne], [http://www.journaldemontreal.com/2013/02/19/la-bonne-education].

${ }^{54}$ Collège néo-classique [site Web], [http://www.collegeneoclassique.org] (10 décembre 2013). 